\title{
E o Passado é uma Roupa que Não nos Serve Mais: uma Reflexão sobre Integração Ensino- Saúde-Comunidade em Curso Médico do Nordeste
}

\author{
And the Past is a Garment that no Longer \\ Suits us: Reflecting on Teaching-Health- \\ Community Integration in a Medical Course \\ in Northeast Brazil
}

Carlos Dornels Freire de Souza $a^{\text {Iอ }}$ Divanise Suruagy Correia ${ }^{10}$ Maria Deysiane Porto Araújo ${ }^{I \oplus}$ Roberta Albuquerque Wanderley ${ }^{1 \odot}$ Michael Ferreira Machado ${ }^{I \oplus}$

\section{PALAVRAS-CHAVE}

- Educação Médica.

- Flexibilidade de Aprendizado.

- Currículo.

- Educação Superior.

\section{RESUMO}

A formação médica, assim como a sociedade brasileira, tem passado por importantes transformações. Tais mudanças buscam atender as demandas sociais e sanitárias contemporâneas, sobretudo no que diz respeito a uma formação voltada à realidade sociossanitária local. Em razão disso, a formação deve buscar desenvolver competências, habilidades e atitudes em um cenário de integração do ensino com a saúde e a comunidade. O curso de Medicina da Universidade Federal de Alagoas, campus Arapiraca, iniciado no ano de 2015, resulta do processo de expansão das escolas médicas do País e do processo de interiorização das ações dessa universidade. O curso está estruturado em três eixos (tutoria, habilidades médicas e integração ensino-saúde-comunidade). Neste texto, pretende-se apresentar o processo de trabalho do eixo de Integração Ensino-Saúde-Comunidade (Iesc), seus principais avanços e os desafios envolvidos no processo de formação. O Iesc tem como missão promover a aproximação entre o acadêmico e a realidade sociossanitária local a fim de garantir uma formação médica capaz de oferecer uma assistência integral, respeitosa, ética, crítica e humanística, considerando o sujeito e o contexto em que está inserido, sua cultura, crença, hábitos e costumes. Em 2017, para cumprir sua missão, o eixo reestruturou o currículo do curso de modo que a formação ocorra no ciclo básico (primeiro e segundo anos), passando pelo ciclo intermediário (terceiro e quarto anos) e alcançando o internato (quinto e sexto anos). Nossa experiência tem mostrado que o aprendizado significativo deve nascer da prática, isto é, do processo de experimentações com a realidade. É nítido o maior envolvimento dos nossos estudantes quando incluímos atividades com finalidades práticas bem definidas. Nossa sistemática de trabalho envolve reuniões mensais, quando são discutidos os principais problemas e elaboradas estratégias de condução do eixo, padronização de cadernos de curso e estratégias avaliativas, processo de formação continuada dos docentes, como parte do plano bianual de qualificação e inclusão de avaliação por parte dos acadêmicos, com o semestre em curso. A variedade de estratégias pedagógicas, todas ancoradas nas metodologias ativas de ensino-aprendizagem, contribui para o maior envolvimento dos nossos estudantes nas aulas. Os principais desafios são consolidar a inserção na rede de saúde local e qualificar o corpo docente no uso de metodologias ativas em todos os processos de aprendizagem. 


\section{KEY-WORDS}

- Medical Education.

- Flexible Learning.

- Curriculum.

- Higher Education.

Recebido em: 05/11/2019

Aceito em: 06/11/2019

\section{ABSTRACT}

Both medical education and Brazilian society as a whole have undergone significant transformations in recent years. These changes seek to meet contemporary social and sanitary demands, especially with regard to a training focused on the local socio-sanitary reality. Medical training should therefore seek to develop skills, abilities and attitudes in a setting where teaching is integrated with health care and the community. The medical course of the Federal University of Alagoas, Arapiraca campus, was founded in 2015, resulting from the expansion of medical schools in Brazil and the internalization of the University's actions. The course is structured on three axes (tutoring, medical skills and teachinghealth-community integration). This text intends to present the work process of the Teaching-HealthCommunity Integration (LESC) axis, its main advances and the challenges involved in the training process. The mission of LESC is to place the academic into closer contact with the local socio-sanitary reality in order to ensure a medical training that offers comprehensive, respectful, ethical, critical and humanistic care, considering the subject and his or her context, culture, beliefs, habits and customs. To fulfill this mission, in 2017 the course curriculum was restructured so as to split the training into the basic cycle ( $1^{\text {st }}$ and $2^{\text {nd }}$ years $)$, the intermediate cycle ( $3^{\text {rd }}$ and $4^{\text {th }}$ years $)$ and, finally, medical internship $\left(5^{\text {th }}\right.$ and $6^{\text {th }}$ years). Our experience has shown that meaningful learning must come from practice, that is, from the process of experimentation with reality. Our students are distinctly more involved and interested when we include activities with well-defined practical purposes. Our work system involves monthly meetings, where major issues are discussed and strategies developed for conducting LESC, the standardization of course books and evaluation strategies, a continuing education program for teachers, as part of the biannual qualification plan and the inclusion of student-led evaluation during the semester. The variety of teaching strategies, all anchored in active learning methodologies, encourages greater involvement of our students in class. The main challenges can be summarised as consolidating student insertion into the local health care network and qualifying the teachers in their use of active methodologies in all learning processes.

\section{INTRODUÇÃO}

A formação médica, resguardadas as devidas proporções e acompanhando a evolução da sociedade brasileira, passou por importantes transformações, iniciadas no século XX e ainda em curso nestas primeiras décadas do novo século. A Conferência Internacional sobre Cuidados Primários em Saúde, ocorrida em 1978, em Alma-Ata, no Cazaquistão, foi a mais importante força propulsora de mudanças no campo da saúde, uma vez que posicionou a lupa do sistema de saúde sobre a atenção primária, alicerce de sustentação e principal porta de entrada do novo processo de trabalho em saúde .

Em terras tupiniquins, na década de 1970, a população experimentava os anos mais cinzentos de sua história: a ditadura militar. Foi nesse período que dois movimentos surgiram e passaram a caminhar lado a lado contra a repressão e a ausência de direitos civis. O primeiro foi o processo de luta contra o poder instituído via golpe militar; já o segundo buscava a saúde como direito social - a chamada reforma sanitária ${ }^{2}$. Os resultados foram o fim da ditadura militar, o restabelecimento da democracia, a promulgação da Constituição cidadã de 1988 e a garantia da saúde como um direito de todos e dever do Estado, dando origem ao Sistema Único de Saúde (SUS), alicerçado nos princípios filosóficos da universidade, integralidade e equidade. Anos depois, nasceu o Programa de Saúde da Família - atual Estratégia Saúde da Família -, buscando efetivar a atenção primária como o alicerce do $\mathrm{SUS}^{3}$.

Em virtude dessas intensas mudanças, o modelo de formação médica vigente à época não mais conseguiria ofertar profissionais capazes de lidar com as novas demandas sociais e sanitárias. O profissional médico assume um novo papel social, o que exige modificação do processo formativo, uma vez que esse novo perfil se assenta na necessidade de transformar a realidade sociossanitária local.

[E o passado é uma roupa que não nos serve mais]. 
Sabia-se que era necessário mudar o processo formativo, mas como? Que ações as escolas médicas deveriam adotar para formar esses novos profissionais? Que metodologias de ensino utilizar? Que competências, habilidades e atitudes deveriam ser valorizadas? Em busca de respostas, muitas experiências foram realizadas nos últimos anos, culminando num conjunto de diretrizes e guias amplamente utilizado nos cursos de Medicina. As dimensões geográficas do País e, portanto, sua heterogeneidade social, política, econômica e cultural demandaram e demandam processos formativos que estejam em sintonia com a realidade local.

Esses antecedentes serviram de base para a nossa experiência no jovem curso de Medicina da Universidade Federal de Alagoas (Ufal), campus Arapiraca, criado em 2015 por meio do processo de interiorização de ações da Ufal e da expansão dos cursos de Medicina no Brasil. Assim, nossa escola médica buscou construir um percurso formativo em três grandes eixos: Eixo Tutorial, Habilidades Médicas e Integração Ensino-Saúde-Comunidade (Iesc).

\section{EIXO DE INTEGRAÇÃO ENSINO-SAÚDE- COMUNIDADE (IESC)}

O Iesc tem como missão "promover a aproximação do acadêmico com a realidade sociossanitária local a fim de garantir uma formação médica capaz de oferecer uma assistência integral, respeitosa, ética, crítica e humanística, considerando o sujeito e o contexto no qual está inserido, sua cultura, sua crença, seus hábitos e seus costumes". A opção pelo termo "saúde" na denominação do eixo - e não "serviço", como geralmente adotado em outros cursos - deve-se a nossa compreensão de que o serviço é uma dimensão do processo saúde-doença. Advogamos que adotar o termo "serviço" seria um reducionismo, tendo em vista que "saúde" e "doença" são resultados dos processos de inserção dos sujeitos no contexto social e, portanto, um produto do meio, suas dinâmicas e tessituras.

Em 2017, para cumprir sua missão, o eixo reestruturou o currículo do curso de modo que a formação ocorra no ciclo básico (primeiro e segundo anos), passando pelo ciclo intermediário (terceiro e quarto anos) e alcançando o internato (quinto e sexto anos) (Figura 1). A arquitetura lógica adotada tem como base as Diretrizes Curriculares Nacionais do curso de graduação em Medicina (Resolução nº 3, de 20 de junho de 2014) ( $^{4}$ e currículo de competências da Sociedade Brasileira de Medicina de Família e Comunidade (SBMFC) ${ }^{5}$, respeitando-se o perfil sanitário loco-regional. A equipe docente é composta por profissionais de diferentes áreas do conhecimento, objetivando um trabalho interprofissional: quatro médicos de família e comunidade, dois psicólogos (um sanitarista e um clínico), um fisioterapeuta (epidemiologista), uma enfermeira, um filósofo, além de docentes substitutos. Novos concursos estão sendo realizados com a intenção de ampliar a equipe.

Figura 1

Arquitetura lógico-formativa do eixo de Integração Ensino-Saúde-Comunidade (IESC) do curso de Medicina da Universidade Federal de Alagoas, campus Arapiraca

\begin{tabular}{|c|c|c|}
\hline $\begin{array}{c}10 \text { período } \\
\text { INTRODUÇÃO À CLÍNICA AMPLIADA }\end{array}$ & $\begin{array}{l}\text { 20 período } \\
\text { IESC } 1\end{array}$ & $\begin{array}{l}\text { 30 período } \\
\text { IESC } 2\end{array}$ \\
\hline $\begin{array}{l}\text { Introdução à Clínica Ampliada no SUS; } \\
\text { Introdução à Medicina de Família e } \\
\text { Comunidade; } \\
\text { Territorialização e diagnóstico comunitário. }\end{array}$ & $\begin{array}{c}\text { Epidemiologia; } \\
\text { Bioestatística; } \\
\text { Produção da informação para a tomada de } \\
\text { decisão em saúde. }\end{array}$ & $\begin{array}{l}\text { Educação em Saúde; } \\
\text { Comunicação em saúde; } \\
\text { Promoção da saúde. }\end{array}$ \\
\hline $\begin{array}{l}\text { 40 período } \\
\text { IESC } 3\end{array}$ & $\begin{array}{l}\text { 5o período } \\
\text { IESC } 4\end{array}$ & $\begin{array}{l}\text { 60 período } \\
\text { IESC } 5\end{array}$ \\
\hline $\begin{array}{l}\text { Abordagem Familiar: Diagnóstico, } \\
\text { instrumentos e práticas. }\end{array}$ & Vigilância em Saúde. & $\begin{array}{l}\text { Gestão da Clínica; } \\
\text { Gestão de serviços de saúde; } \\
\text { Planejamento em saúde. }\end{array}$ \\
\hline $\begin{array}{l}\text { 7º período } \\
\text { IESC } 6\end{array}$ & $\begin{array}{l}\text { 8 período } \\
\text { IESC } 7\end{array}$ & 9 período \\
\hline Psicologia médica. & Saúde do trabalhador. & $\begin{array}{l}\text { Internato em Saúde da Família e Saúde } \\
\text { Coletiva }\end{array}$ \\
\hline
\end{tabular}




\section{AÇÕES DE APROXIMAÇÃO COM A COMUNIDADE E APRENDIZADO ORIENTADO POR RESULTADOS}

As ações desenvolvidas nos últimos dois anos de trabalho mostram o empenho da equipe docente em promover a aproximação entre o acadêmico de Medicina e a realidade sociossanitária do município, enfatizando a participação ativa do discente na construção do conhecimento e no desenvolvimento de habilidades, atitudes, valores e competências que possam transformar a realidade local. Em cada período/semestre são definidos produtos finais, denominados "produtos de consolidação do saber", conforme exemplificado no Quadro 1.

\section{QuAdro 1}

Produtos de consolidação do saber adotados pelo eixo de Integração Ensino-Saúde-Comunidade

(Iesc) do curso de Medicina da Universidade Federal de Alagoas, campus Arapiraca

$\begin{array}{cc}\text { Período } & \begin{array}{c}\text { Produto de consolidação do saber } \\ \text { Diagnóstico comunitário }\end{array} \\ 1^{\circ} & \begin{array}{r}\text { Exposição fotográfica sobre o diagnóstico comunitário } \\ \text { Boletim epidemiológico e pesquisa científica }\end{array} \\ 2^{\circ} & \text { Vídeos educativos para salas de espera } \\ 3^{\circ} & \text { Formação sobre instrumento de diagnóstico familiar } \\ 4^{\circ} & \text { Curso de vigilância para agentes comunitários de saúde } \\ 5^{\circ} & \text { Eventrução de planos de regionalização } \\ 6^{o} & \text { Eventífico } \\ 7^{o} & \text { Cartilhas educativas com temas relacionados à saúde } \\ \text { do trabalhador }\end{array}$

Nossa experiência tem mostrado que o aprendizado significativo deve nascer da prática, isto é, do processo de experimentações da realidade. É perceptível o maior envolvimento dos nossos estudantes quando incluímos atividades com finalidades práticas bem definidas. São exemplos: (a) no ensino de bioestatística, os acadêmicos coletam dados e analisam a qualidade de vida dos próprios estudantes do curso, de modo que todas as aulas são práticas e orientadas por um resultado, posteriormente apresentado em evento; (b) no módulo de gestão, as atividades são orientadas para a compreensão do plano de regionalização do estado e a construção simulada de planos regionais integrados; (c) em territorialização, além do diagnóstico comunitário construído com o apoio da unidade básica de saúde, os resultados são apresentados por meio de exposição fotográfica, posteriormente transformada em produção científica. Por meio desse processo de trabalho, nossos acadêmicos têm conquistado prêmios em eventos científicos, tanto regionais quanto nacionais, e publicações em revistas especializadas.

Além dos produtos definidos e do ensino orientado por resultados, há de se destacar o processo de curricularização de extensão, incluído no projeto pedagógico do curso. Esse foi um compromisso assumido pelo curso para fortalecer as ações "extramuros", auxiliando os serviços de saúde locais a reduzir os vazios assistenciais, além de democratizar o conhecimento produzido no âmbito do curso. Essas ações envolvem os três eixos do curso de Medicina.

\section{PLANEJAMENTO E AVALIAÇÃO DO PROCESSO DE TRABALHO}

Nossa sistemática de trabalho envolve: (a) reuniões mensais, quando são discutidos os principais problemas e elaboradas estratégias de condução do eixo; (b) padronização de cadernos de curso e estratégias avaliativas; (c) processo de formação continuada dos docentes, como parte do plano bianual de qualificação; (d) inclusão de avaliação por parte dos acadêmicos, com o semestre em curso (três avaliações - no final do primeiro mês de aula, no meio e no final do semestre). Esse último componente foi recentemente incluído com a finalidade de monitorar o semestre letivo em curso. Nelas, é possível identificar problemas em tempo oportuno e estabelecer estratégias de intervenção com vistas a sanar alguma dificuldade.

\section{METODOLOGIAS DE ENSINO}

A variedade de metodologias de ensino contribui para o maior envolvimento dos nossos estudantes nas aulas: oficinas de trabalho (OT), aprendizagem baseada em projetos, pesquisa e produção do conhecimento, palestras/conferências/seminários, estudo individual e dirigido, atividades em campo, cineviagem educacional, Fishbowl, Team Based Learning (TBL), painel integrado, círculo de cultura, situação-problema, narrativas, simulações em espaço protegido e jogos educativos.

Todos esses recursos são utilizados com vistas a centrar no estudante o processo de aprendizagem, levando em consideração desde a competência cultural de cada um até a interface com os saberes apresentados e debatidos na universidade, sem desconsiderar o perfil socioepidemiológico local.

\section{OS DESAFIOS AINDA SÃO MUITOS}

A implantação de uma escola médica no interior do Nordeste brasileiro compreende um processo de articulação que envolve aspectos políticos, culturais e organizacionais que potencializam ou dificultam a efetivação de práticas inovadoras na formação médica. Em um curso tão jovem e ainda em formação, como o nosso, são muitos os desafios a superar. O primeiro desafio que destacamos é consolidar a inserção dos alunos na rede de saúde local.

O curso funciona numa cidade de mais de 230 mil habitantes com cobertura da Estratégia Saúde da Família em 97,86\% 
da população. Essa alta taxa proporciona um contexto favorável ao desenvolvimento de ações na rede de atenção básica ${ }^{6}$. Entretanto, todos os cenários de práticas utilizados no curso são administrados por entes públicos (Prefeitura Municipal de Arapiraca e Estado de Alagoas) ou instituições particulares, em especial filantrópicas, conveniadas ao SUS. Nenhum dos equipamentos de saúde é gerido ou cogerenciado pela Universidade Federal de Alagoas, o que representa uma barreira em face dos trâmites existentes para tomadas de decisão e gestão destes serviços, que não foram inicialmente estruturados para funcionar como serviços-escola.

O segundo desafio diz respeito à utilização de metodologias ativas em todo o processo de ensino-aprendizagem. O uso dessas estratégias metodológicas requer uma potência criativa do docente para propor aos alunos ações que se distanciem da visão tradicional, descentrando do professor o controle do currículo e os modos de ensinar. A problemática está na produção de docentes inseridos e comprometidos com essa proposta pedagógica, pautados nessa construção coletiva e democrática do saber.

É preciso aceitar o desafio de formar profissionais médicos comprometidos com a realidade sociossanitária local, com a inserção, desde o primeiro dia de aula, nos serviços de saúde da atenção básica, possibilitando-lhes compreender das vicissitudes dos territórios aos instrumentos de gestão na saúde. O médico egresso desse processo formativo, baseado na interprofissionalidade e no pluralismo, é um profissional com competências e habilidades nas dimensões ética (compromisso com o outro), estética (potência criativa em face das mais variadas situações presentes no cotidiano dos profissionais de saúde) e política (perspectiva da transformação da sociedade).

\section{REFERÊNCIAS}

1. Mendes IAC. Desenvolvimento e saúde: a declaração de Alma-Ata e movimentos posteriores. Rev. Latino-Am. Enfermagem. 2004; 12(3): 447-48.

2. Escorel S. História das políticas de saúde no Brasil de 1964 a 1990: do golpe militar à reforma sanitária. In: Giovanella L. et al. (Org.). Políticas e sistemas de saúde no Brasil. Rio de Janeiro: Fiocruz, 2008.
3. Sousa CDF. Programa Saúde da Família - PSF: a ação do estado, o cenário de implantação e a importância do território para a sua efetivação. Hygeia. 2014; 10(18): 207-21.

4. Brasil. Ministério da Educação. Diretrizes Curriculares Nacionais do Curso de Graduação em Medicina [acesso 2019 agost 28]. Disponível em: http://portal.mec.gov.br/cne/ arquivos/pdf/Med.pdf

5. Sociedade Brasileira de Medicina de Família e Comunidade. Currículo Baseado em Competências para Medicina de Família e Comunidade. 2015; 66-8. [acesso 2019 agost10]. Disponível em: http://www.sbmfc.org.br/media/Curriculo Baseado em Competências (1).pdf

6. Cobertura da atenção básica [Internet]. 2019 [acesso 2019 agost 29]. Disponível em: https://egestorab.saude.gov. br/paginas/acessoPublico/relatorios/relHistoricoCoberturaAB.xhtml

\section{CONTRIBUIÇÃO DOS AUTORES}

Souza CDF, Correia DS, Araújo MDP, Wanderley RA, Machado MF participaram da concepção, delineamento, análise e interpretação dos dados, escrita do manuscrito e revisão crítica do conteúdo. Todos os autores aprovam a versão final a ser publicada.

\section{CONFLITO DE INTERESSES}

Sem conflitos de interesses

\section{ENDEREÇO PARA CORRESPONDÊNCIA}

Carlos Dornels Freire de Souza

Universidade Federal de Alagoas

Departamento de Medicina

Rodovia AL-115, s/n ${ }^{\circ}$

Bom Sucesso - Arapiraca

CEP 57309-005 - AL

E-mail:carlos.freire@arapiraca.ufal.br 